\title{
Evaluating a new methodology for providing individualized feedback in healthcare on quality of life and its importance, using the WHOQOL-BREF in a community population.
}

\section{INTRODUCTION}

Research on patient-reported outcome measures shows it is possible to use quality of life (QoL) information from individual patients to improve the quality of clinical communications [1-6], and jointly identify goals and priorities for future health and health care [7]. Many patients actively want to tell health professionals (HPs) about their QoL [1,8,9] and the NHS Operating Framework proposes putting 'patients at the centre of decision-making with their experience of health' [10]. As QoL life measures offer accurate and expedient tools for the rapid collection of reliable, valid information about individual subjective QoL, some HPs value access to it [2,11,12]. However not all welcome using QoL assessment in routine clinical practice, citing organisational, personal, practical, financial, educational and occupational reasons [13]. For instance, not all HPs understand how to interpret QoL information [13]. A more serious barrier to implementation is where HPs 'know' their patients and their QoL well, and see assessment as irrelevant, time consuming, and even a threat to their jobs [14]. Reassuringly, appropriate training can successfully address many of these challenges [15].

Resistance among HPs led us to take a different approach in the present research. We argue if the general population can utilize user-friendly guidance to satisfactorily understand and interpret their own QoL results, then assistance from HPs may be unnecessary, except where major health problems (e.g. pain, disability) exist. If a profile of results is readily understandable unaided, there would be financial savings to services, as HPs would not need to conduct this work routinely. Yet with patient permission, health and social care professionals could still gain access to independently completed information on request. For individuals, completing QoL measures and owning their personal results can promote wellbeing $[4,16,17]$. In this way, QoL feedback could serve a health promotion and disease prevention role, which has been scarcely appreciated. Furthermore, if community populations can understand how to interpret their QoL results with suitable guidance, then HPs should also be able to understand this information. Nevertheless, the individual is best placed to interpret their own subjective QoL results, as only they will fully comprehend them within the complex context of their experience.

Our unique individual approach draws on principles previously tested in group data from surveys of sick and well people in 15 countries $(n=4,800)$ world-wide, where compatible WHOQOL core QoL and Importance measures were administered [18]. Results showed that the very poorest QoL could be identified where a QoL dimension was both poor and very important. As such, the dimension is perceived to be even poorer because of its high importance. For full clarification, see Skevington \& O’Connell [18]. Presenting results from both measures together not only allowed poor and good QoL to be readily identified, but also enabled poor QoL areas to be prioritized and targeted for improvement. 
Based on these principles, we have developed a new type of guided individualized feedback presenting importance information alongside core QoL scores for individual appraisal [19]. As previously reported, we used pre- and post-intervention measures to quantitatively test the effects of this novel type of feedback on the wellbeing of a community sample containing well and sick people [17]. Presented here, the study also used subjective evaluations of the feedback, and follow-up interviews with those who were positive about it, with the explicit aim of capturing constructive views about how the feedback might be used, and its relevance and value during healthcare.

Our research is grounded in Self-Regulation Theory (SRT) [20,21] which asserts that individuals use feedback information to adapt to, or steer towards their goal(s). This is relevant here because goal achievement is integral to the World Health Organization's (WHO) QoL definition, as it is used to judge QoL: 'An individuals' perceptions of their position in life, in the context of the culture and value systems in which they live, and in relation to their goals, expectations, standards and concerns.' [22].

\section{METHODS}

Participants: Participants were purposively recruited from the community, primary care and charities. Quotas were used for chronic illness (70\%) and health (30\%) [17,19]. Advertisements inviting participation were placed on the websites of the Multiple Sclerosis Society, Asthma UK, National Eczema Society, Epilepsy Action, British Lung Foundation, and posted on a university campus. A General Practitioner invited patients registered in NHS Primary Care, to participate. University Department and local NHS Research Ethics Committees gave their approval ${ }^{1}$. Participants were assured of anonymity and confidentiality and gave fully informed written consent. They were included if over 18 years, and excluded if they were not literate and fluent in English; a necessary condition for independent completion of questionnaires. This was assessed through written communications and telephone conversations.

Procedure: Participants assessed their QoL by completing the WHOQOL-BREF [23] and abbreviated WHOQOL-Importance scales [18]. They received individualised graphical profiles of their results (an example extract is shown in Figure 1). Guided feedback on how to read and interpret the profile was provided either a) during a telephone call from a researcher using a prepared script, or b) by printed instructions [19]. Protocols for both conditions were matched in terms of the steps participants should follow to interpret their results. Prior testing established the feasibility and acceptability of each condition, and detected no differences between conditions in post-feedback outcome measures, indicating equivalence of effect [17,19]. During feedback, the range of possible facet scores (1-5) was clearly stated ( 0 was included on the graph scales to show missing items). Participants firstly inspected their core QoL scores to identify good QoL (>3.0). They then found any large differences between Importance and core QoL, focusing on where scores specifically indicated poor QoL $(<3.0)$, and high importance $(>3.0)$. For each area they were invited to consider what they might do to make large gaps smaller. For ethical reasons, the final instruction asked them to identify facets where QoL and Importance were both high, to end the intervention positively. No normative information was offered.

\footnotetext{
${ }^{1}$ Gathering exact age data was not approved by the NHS Ethics committee.
} 
[INSERT FIGURE 1 HERE]

\section{Assessment:}

a. Post Feedback Evaluation Questionnaire

Immediately after receiving their QoL feedback, participants completed a paper-based, post-feedback evaluation of change questionnaire, inquiring about their experience of the process. An 8-point Likert scale evaluated how much their QoL had changed overall as a result of the QoL feedback $(0=$ much worse to $7=$ much better $)$. Eight-point Likert scales $(0=$ not at all to $7=$ completely $)$ asked them to evaluate how much: (a) the way they thought about their QoL had subsequently changed; (b) their plans or expectations for the future had changed; (c) each QoL domain had changed (physical; psychological; social; environment). Explanatory comments were requested for each change rating. They were asked how helpful they expected their experience of feedback would be in the short, medium and long-term, using separate 8 -point scales $(0=$ very unhelpful to $7=$ very helpful $)$.

\section{b. Follow-up Interviews}

We invited participants to volunteer to take part in further research and selectively invited to a followup interview those who reported that the feedback process was helpful, to constructively explore how this information might be profitably used and by whom.

Semi-structured telephone interviews employed a mixed-methods approach; namely open-ended and multi-choice questions. Questions asked about subsequent appraisals, and any additional use of the feedback graphs. They were designed to facilitate disclosures about how QoL feedback might be used including by, or with a HP. Prompts e.g. “why?”, “why not?”, “what did you conclude?”, explored initial responses. Interviews were audiotaped with prior consent.

\section{Data Analysis:}

a. Post Feedback Evaluation Questionnaire

Categorical response frequencies for evaluations were recorded, and recoded to create useful dichotomies e.g. change vs. no change. Following Donovan and Saunders [24], explanatory comments captured by the evaluation questions were transcribed by question. Replies were analysed in stages: interpreting the general sense of statements; identifying themes; organising themes into a framework, and coding data according to the framework. Coding was independently checked by a second researcher for reliability, and adjustments made.

\section{b. Follow-up Interviews}

Interviews were transcribed verbatim into NVivo (version 9). Transcripts were coded for each question using a pragmatic framework: the perceived value of the process of completing a QoL instrument; value attached to the feedback; and uses of the QoL profile, including by professionals. Interviews were conducted until data saturation was achieved. 


\section{RESULTS}

Samples:

Expressions of interest were received from 207 eligible adults. Eighty \% $(\mathrm{n}=165)$ subsequently entered the study, completed the WHOQOL-BREF and WHOQOL-Importance questionnaires, and received feedback. Seventy-eight $\%$ of these $(n=129)$ completed and returned the post-feedback evaluation questionnaire: $66 \%$ women, $64 \%$ tertiary educated, $96 \%$ white and $76 \%$ living with a spouse, partner or family. Twenty-eight $\%$ were retired, $34 \%$ employed full-time, and $12 \%$ part-time. Evaluation responders represented a broad cross-section of ages (see Table 1).

Purposive recruitment provided a heterogeneous sample across chronic physical illnesses/conditions and health. Chronic diseases $(n=85)(66 \%)$ were classified by the International Classification of Diseases [25] and included musculoskeletal (21\%), respiratory (19\%), nervous system (19\%), circulation (13\%), dermatology (11\%), endocrine /metabolic (8\%), and other (9\%) conditions.

\section{[INSERT TABLE 1 HERE]}

We identified a pool of 21 for follow-up interview. This subset satisfied both positivity criteria of volunteering for further research, and evaluating the feedback as potentially helpful. Thirteen agreed to an in-depth interview; all were $>45$ years, about half $(\mathrm{n}=7)$ were female, and most $(\mathrm{n}=11)$ previously reported an illness.

\section{Post feedback evaluation questionnaire:}

a. Evaluating change

Figure 2 shows a substantial $65 \%$ of respondents reported they thought differently about their QoL following feedback. Forty \% reported their psychological health had altered, and $34 \%$ thought it had changed their planning and expectations for the future. No association was detected between selfperceived changes and whether or not participants reported a chronic illness, indicating that reporting change was independent of health status. Fourteen \% reported they felt their overall QoL had improved and $84 \%$ reported it had remained about the same.

\section{[INSERT FIGURE 2 HERE]}

b. Qualitative findings

Illustrated below, the main themes were: better understanding of QoL and its importance; selfaffirmation; helpfulness of feedback; changing goals and expectations; and motivation to change behaviour.

Data indicated that after feedback, participants believed they had a better understanding of QoL

and/or changed views about what aspects were important. This was equally true for healthy and ill. 
Raising self-awareness about which aspects of QoL were important helped them to redirect their life e.g. putting trivia into relief. Some said they would pay greater attention to important aspects of life, now they had recognized them. Occasionally important issues were viewed negatively e.g. where work was excessively important, indicating an imbalanced life. Importance was a recurring theme across the quotes, highlighting its significance.

\footnotetext{
"It drew my attention to some aspects of my health that are important but I had failed to recognize their importance until now." (female, ill, aged 55-64)
}

\begin{abstract}
"Seeing the areas in black and white and how important they are to me personally, has made me look at those aspects of my own quality of life in a different way." (healthy female, aged
\end{abstract} $35-44)$

"It has raised my awareness of thinking about how important certain aspects are to me." (healthy female, aged 18-24)

Insights were interpreted as self-affirming and helpful, particularly where participants' appreciated that the feedback was consistent with their self-perceptions. This underscores the face validity of the intervention.

“A helpful and thought-provoking analysis, but mainly reassuring...” (healthy male, aged 35 44)

"It's good to see where I am scoring well, as well as the areas where I already knew I was scoring badly. " (female, ill, aged 18-24)

"It is interesting, and lucky for me, to see that the areas of my life are roughly in line with the importance I give them." (healthy female, aged 55-64)

As shown in Figure 2, 34\% of participants reported their goals and expectations had altered as a result of the feedback, and comments often indicated increased motivation to bring about positive changes in behaviour. Feedback had facilitated the identification of specific QoL areas that people were committed to improve. The relative proportions of healthy and ill participants who made these comments were consistent with the overall sample.

\footnotetext{
"[The study] has underlined some areas that I was aware of but can now concentrate on doing something about them." (female, ill, aged 45-54)

"I will be taking more time to look at where the problems lie in my life and how I can go about changing them to make me feel better." (female, ill, aged 18-24)
} 
“... helps me to identify and isolate areas in need of improvement. This is great because rather than having the insurmountable task of 'trying to make my life better', I can work on specific areas with specific actions. " (male, ill, aged 25-34)

c. Evaluating the helpfulness of QoL feedback

Of the responses from 129 participants, over 50\% expressed a belief the QoL feedback they had received would prove helpful in the next 3 months, and beyond (see Table 2). There was no association between reported helpfulness and whether participants were healthy or ill.

\section{[INSERT TABLE 2 HERE]}

\section{Follow-up interviews}

a. Pragmatic reflections on the process of quality of life feedback

Follow-up data indicated an appreciation of the insights gained as a result of completing both

WHOQOL questionnaires, by ill and healthy participants.

"I felt at the beginning of the questionnaire and towards the end of the questionnaire and all the questions that were asked, I felt that I was getting a picture of who I am, what I am, and I could use that for the future." (male, ill, aged 75+)

"It gave me a different perspective on things that were happening or have happened, or experiences I've had and it just made me think about them a little more sensibly and in a linked fashion." (healthy male, aged 75+)

"I think it can help make a difference because it makes you face it. You don't sit down normally every day and think about the things that are on that graph. So maybe it just focuses you." (female, ill, aged 45-54)

b. Sharing quality of life information with a health professional

Quotations showed all interviewees expected it would be useful to share this QoL information with a HP. Principally, they believed it would enhance HP's understanding of the patients' QoL, especially physical health, psychological state, and lifestyle:

"Well I think it is because I think that is a good indication of how fit you are and your view on how you treat any illnesses." (female, ill, aged 65-74)

"Well presumably the health professional... would be able to assess whether I was stressed, over-stressed, or whatever, by the situation I'm in". (female, ill, aged 70+) 
"I think in my case, the way I operate, I think [the feedback] would be more useful for me to discuss with a health professional than a spouse or friend or partner." (healthy male, aged $75+$ )

Participants suggested facilitating HP's understanding of their QoL might assist with diagnosing health problems. They welcomed employing this information as the basis for individualised positive health advice, irrespective of whether they were ill or healthy:

“... say I went along to see him with a problem, and he had that information there, he might be able to use it, you know, to assist in making the decision on what the problem might be." (male, ill, aged 65-74)

“... I think it would give the doctor a baseline... and if they can't articulate it, if they can't pinpoint what the problem is, it would help to sort of narrow down the areas." (female, ill, aged 45-54)

“...if you've got something in front of you that they have filled in, that says actually this is your life ... and especially if there were options, you know, sort of "there's a big gap here"... I don't know... "in our experience there are five routes out of this". (female, ill, aged 55-64)

"Obviously if any there's a diagnosis of anything you had, it would help, because your lifestyle is important. How you live is to your health really. And that's where I think the doctor would come into that equation." (healthy male, aged 65-74)

One participant said a better understanding of QoL could challenge stereotypes that HPs may hold about groups, (e.g. older people) enabling them to be treated as individuals:

"Well try and think more... stop thinking of my age group as a set thing that's in the past. I don't go back any further music-wise, than Elvis Presley and The Beatles. I don't want to sing Daisy, Daisy... I absolutely refuse!" (female, ill, aged 75+)

However, not all comments were uniformly affirmative:

“... a lot of the things I don't think I could do anything about, or I've probably already done them or decided that I cannot do any more about it." (female, ill, aged 75+)

Sceptical comments from some ill participants highlighted concerns that HPs may be restricted in using QoL feedback in practice: 
"I think it would be useful, but I can't see there ever being enough time for the health professional." (female, ill aged 45-54)

“...maybe there would be more understanding by the professionals of any given situation. But they're probably overworked anyway, so maybe it wouldn't do anything." (female, ill, aged 70+)

\section{DISCUSSION}

The aim of our mixed-methods pilot intervention was to explore individual evaluations of a new type of feedback that enabled people to identify and consider not just their own QoL but also its importance. Conducted in a community and primary care sample, we aimed to find out whether this unique feedback style would be valued by well and sick people, and whether it was seen to be relevant to selfmanaging health or lifestyle, and valuable for healthcare settings. We found that people are capable of using this information themselves with suitable guidance, and see a distinct application to health, through sharing this information with HPs. While our findings show routine assessment by HPs of all patients will not be necessary, some with serious illness will still need QoL assessment for clinical purposes. For this reason, HPs may wish to add the assessment and interpretation of QoL information to their skills set in the foreseeable future. Already QoL information is currently collected in NHS orthopaedic surgery and for some chronic diseases, but its data are used in audit and governance, and not routinely to benefit individual patients.

We found most participants, healthy and chronically ill, reported that the way they had thought about their QoL had changed after this new type of feedback. Revised perceptions of QoL or altered cognitions about the importance of particular aspects were reported, pointing to response shift [26]. The feedback had often been self-affirming so participants felt more positive, or appreciative of particular aspects of their lives.

Reporting changes to their life goals and expectations, participants talked about intentions to improve their QoL and plans to change behaviour, as a direct result of the feedback. Primarily they aimed to focus on specific features and identify actions, and again this was characteristic of healthy and ill. It is arguable that receiving our type of QoL feedback engendered a sense of readiness to take action, irrespective of health status. Behavioural preparedness forms the conceptual basis of many health behaviour change models [27], and future studies should investigate the mechanisms whereby priming through this feedback type contributes to actual change in behaviours.

Chronically ill and healthy participants indicated that QoL feedback would be helpful in the medium and/or longer term, suggesting equal benefit to all, irrespective of health status. Previously we compared WHOQOL-BREF scores following feedback in those helped and not helped by QoL feedback and found those who were not helped reported significantly poorer psychological QoL, but 
not physical QoL [19]. Screening for anxiety and depression or using feedback protocols adjusted to poorer psychological health, may be useful in the future.

Previous findings indicated that most participants welcome QoL advice based on empirical results. Patients want to talk about their QoL to a clinician, and expect them to understand and take account of factors influencing their QoL $[1,9,8]$. Our study confirmed that community samples believe their QoL information will help HPs to treat them with greater understanding. However they also want HPs to use this information to provide tailored, targeted, support and guidance. Requests for guidance may in part, reflect an underlying predisposition to hand responsibility for action to an external authoritative source, or arise from uncertainty about what action to take within an information void. Such issues offer interesting new lines of investigation.

Implementing routine individual QoL feedback in healthcare pathways raises questions about the training of HPs, and addressing resistance arising from threats to professional identity. Ill participants also underscored known concerns that time pressure would impede HPs' use of this information in consultations [28]. Nevertheless we offer a generic protocol whereby QoL feedback could be used by the whole health and social care team across a comprehensive range of issues. However if this feedback is administered by HPs, it may come to represent an intervention that is "provided" rather than an approach to support self-management of lifestyle. It may therefore be more appropriate to focus on encouraging the general population to self-administer the feedback routinely before a consultation, and to offer it to professionals who assist them. This could support the HP's aim of enabling mutual decision-making. Focusing on self-management would have economic benefits of reducing financial and time costs associated with staff administration.

While our findings indicate opportunities to apply QoL feedback, they are consistent with current equivocal conclusions about its effect on wellbeing. While some studies find no effects [2,29,3], others provide limited evidence of improved wellbeing $[4,16,11]$. It is arguable that understanding one's own QoL does not necessarily lead to change at the time it is received, and the majority of intentions to adopt new behaviours are not always followed by actual behaviour change [30]. It is plausible, however, that QoL feedback may prime readiness to take action, and this type of behavioural preparedness underlies many health behaviour models [27].

It is noteworthy that post-feedback changes reported in the present study reflect subjective evaluations, rather than measurable differences [17], and should therefore be interpreted with caution. We also recommend that future research explores the potential "attention" effect provided by this feedback protocol by comparing results of those who complete the QoL measures with and without receiving their results. Other avenues of investigation would be to determine the optimum length of time delay between QoL feedback and its' evaluation, and to establish the longevity of feedback effects. This additional knowledge would assist in the practical development of feedback intervention protocols for a randomized controlled trial. 
The follow-up interview sample in our study was small and deliberately selective as our aim was to gather constructive suggestions for the application of QoL feedback, not to present a balanced account from mixed evaluations. However a larger and representative sample would be of value in further research, and helpful in examining generalizability.

Our novel approach used graphical profiles to simultaneously present the importance of each dimension alongside individual self-ratings of QoL and our findings offer insights into opportunities for applying QoL feedback. Our research draws on studies about the importance of QoL to groups [3135], showing how combining this information offered an innovative tool that could identify the very poorest QoL [18]. For individuals, this approach enables them to more readily recognise and prioritise QoL aspects for action, and may be helpful to HPs in developing treatment plans. This interpretation was confirmed by our finding that the "importance" of QoL was a recurring theme.

To capitalize on the benefits of individualised, comprehensible guided feedback, this pilot complex intervention needs testing in a fully randomized controlled trial, and additional research is needed into its feasibility and acceptability within healthcare settings. However, our results suggest that it has a potential role beyond self-monitoring and disease management. While it could support routine clinical decision-making, it may also be valuable as a psychological intervention supporting self-management, and wellbeing in community settings.

\section{ACKNOWLEDGEMENTS}

The authors are very grateful to Dr Sanjeev Popli and Mrs Tracy Harris at Jubilee Field Surgery, NHS Wiltshire Clinical Commissioning Group; and to the Multiple Sclerosis (MS) Society; Asthma UK; the National Eczema Society; Epilepsy Action, and the British Lung Foundation for publicising this study.

[Word count: 4000]

\section{COMPLIANCE WITH ETHICAL STANDARDS}

Conflict of Interest: The authors declare that they have no conflicts of interest arising from the research described in this paper.

Ethical approval: All procedures performed in studies involving human participants were in accordance with the ethical standards of the institutional and/or national research committee, and with the 1964 Helsinki declaration and its later amendments, or comparable ethical standards.

Informed consent: Informed consent was obtained from all individual participants included in the study. 


\section{REFERENCES}

1. Detmar, S. B., Muller, M. J., Schornagel, J. H., Wever, L. D. V., \& Aaronson, N. K. (2002). HealthRelated Quality-of-Life Assessments and Patient-Physician Communication: A Randomized Controlled Trial. JAMA, 288(23), 3027-3034.

2. Hilarius, D. L., Kloeg, P. H., Gundy, C. M., \& Aaronson, N. K. (2008). Use of health-related quality-of-life assessments in daily clinical oncology nursing practice. Cancer, 113(3), 628637.

3. Santana, M. J., Feeny, D., Johnson, J. A., McAlister, F. A., Kim, D., Weinkauf, J., et al. (2010). Assessing the use of health-related quality of life measures in the routine clinical care of lungtransplant patients. Quality of Life Research, 19(3), 371-379.

4. Velikova, G., Booth, L., Smith, A. B., Brown, P. M., Lynch, P., Brown, J. M., et al. (2004). Measuring quality of life in routine oncology practice improves communication and patient well-being: a randomized controlled trial. Journal of Clinical Oncology, 22(4), 714-724.

5. Takeuchi, E. E., Keding, A., Awad, N., Hofmann, U., Campbell, L. J., Selby, P. J., et al. (2011). Impact of patient-reported outcomes in oncology: A longitudinal analysis of patient-physician communication. Journal of Clinical Oncology, 29(21), 2910-2917.

6. Velikova, G., Keding, A., Harley, C., Cocks, K., Booth, L., Smith, A. B., et al. (2010). Patients report improvements in continuity of care when quality of life assessments are used routinely in oncology practice: Secondary outcomes of a randomised controlled trial. European Journal of Cancer, 46(13), 2381-2388.

7. Marshall, S., Haywood, K. L., \& Fitzpatrick, R. (2006). Impact of patient-reported outcome measures on routine practice: a structured review. Journal of Evaluation in Clinical Practice, 12, 559-568.

8. Jacobs, J., van de Lisdonk, E., Smeele, I., van Weel, C., \& Grol, R. (2001). Management of patients with asthma and COPD: monitoring quality of life and the relationship to subsequent GP interventions. Family Practice, 18(6), 574-580.

9. Detmar, S. B., Aaronson, N. K., Wever, L. D. V., Muller, M., \& Schornagel, J. H. (2000). How are you feeling? Who wants to know? Patients' and oncologists' preferences for discussing healthrelated quality-of-life issues. Journal of Clinical Oncology, 18(18), 3295-3301.

10. Department of Health (2011). The Operating Framework for the NHS in England 2012/2013. https://www.gov.uk/government/uploads/system/uploads/attachment_data/file/216590/dh_131 428.pdf. Accessed 4.1.13 2013.

11. Gutteling, J. J., Darlington, A.-S. E., Janssen, H. L. A., Duivenvoorden, H. J., Busschbach, J. J. V., \& Man, R. A. (2008). Effectiveness of health-related quality-of-life measurement in clinical practice: a prospective, randomized controlled trial in patients with chronic liver disease and their physicians. Quality of Life Research, 17, 195-205.

12. Bossola, M., Murri, R., Onder, G., Turriziani, A., Fantoni, M., \& Padua, L. (2010). Research Physicians' knowledge of health-related quality of life and perception of its importance in daily clinical practice. Health and Quality of Life Outcomes, 23(8), 43.

13. Skevington, S. M., Day, R., Chisholm, A., \& Trueman, P. (2005). How much do doctors use quality of life information in primary care? Testing the Trans-Theoretical Model of behaviour change. Quality of Life Research, 14, 911-922.

14. Nurbai, M. (2000). Practice nurse perspectives on the use of quality of life information and assessment in routine practice. University of Bath,

15. Santana, M. J., Haverman, L., Absolom, K., Takeuchi, E., Feeny, D., Grootenhuis, M., et al. (2015). Training clinicians in how to use patient-reported outcome measures in routine clinical practice. Quality of Life Research, 24(7), 1707-1718

16. De Wit, M., De Waal, H., Bokma, J. A., Haasnoot, K., Houdijk, M. C., Gemke, R. J., et al. (2008). Monitoring and discussing health-related quality of life in adolescents with type 1 diabetes improve psychosocial well-being - A randomized controlled trial. Diabetes Care, 31(8), 15211526.

17. Llewellyn, A., \& Skevington, S. (2015). Using guided individualised feedback to review selfreported quality of life in health and its importance. Psychology \& Health, 30(3), 301.

18. Skevington, S. M., \& O'Connell, K. A. (2004). Can we identify the poorest quality of life? Assessing the importance of quality of life using the WHOQOL-100. Quality of Life Research, 13(1), 23-34.

19. Llewellyn, A. M. (2012). Understanding quality of life: investigating the effects of individualised feedback on wellbeing. University of Bath, 
20. Carver, C. S., \& Scheier, M. F. (1982). Control theory - a useful conceptual framework for personality-social, clinical and health psychology. Psychological Bulletin, 92(1), 111-135.

21. Carver, C. S., \& Scheier, M. F. (1998). On the self-regulation of behaviour. Cambridge, UK: Cambridge University Press.

22. The WHOQOL Group (1995). The World Health Organization Quality Of Life Assessment (WHOQOL): Position paper from the World Health Organization. Social Science and Medicine, 41(10), 1403-1409.

23. Skevington, S. M., Lotfy, M., \& O'Connell, K. A. (2004). The World Health Organization's WHOQOL-BREF quality of life assessment: Psychometric properties and results of the international field trial. A Report from the WHOQOL Group. Quality of Life Research, 13(2), 299-310.

24. Donovan, J., \& Saunders, C. (2005). Key issues in the analysis of qualitative data in health services research. In A. Bowling, \& S. Ebrahim (Eds.), Handbook of health research methods. Maidenhead, UK: Open University Press.

25. World Health Organization (2010). ICD-10. http://www.who.int/classifications/icd/en/. Accessed 5th February 2010.

26. Sprangers, M. A. G., \& Schwartz, C. E. (1999). Integrating response shift into health-related quality of life research: a theoretical model. Social Science and Medicine, 48(11), 1507-1515.

27. Conner, M., \& Norman, P. (2005). Predicting Health Behaviour: Research and Practice with Social Cognition Models (2nd ed.). Maidenhead, UK: Open University Press

28. Boyce, M. B., Browne, J. P., \& Greenhalgh, J. (2014). The experiences of professionals with using information from patient-reported outcome measures to improve the quality of healthcare: a systematic review of qualitative research. BMJ Quality \& Safety, 23(6), 508-518.

29. Rosenbloom, S. K., Victorson, D. E., Hahn, E. A., Peterman, A. H., \& Cella, D. (2007). Assessment is not enough: A randomized controlled trial of the effects of HRQL assessment on quality of life and satisfaction in oncology clinical practice. Psycho-Oncology, 16(12), 1069-1079.

30. Sheeran, P. (2002). Intention-behavior relations: A conceptual and empirical review. European Review of Social Psychology, 12, 1-36.

31. Baile, W. F., Palmer, J. L., Bruera, E., \& Parker, P. A. (2011). Assessment of palliative care cancer patients' most important concerns. Supportive Care in Cancer, 19(4), 475-481.

32. da Rocha, N. (2011). Evaluation of quality of life and importance given to spirituality/religiousness/personal beliefs (SRPB) in adults with and without chronic health conditions. Revista de psiquiatria clínica, 38(1), 19-23.

33. Molzahn, A., Skevington, S. M., Kalfoss, M., \& Schick Makaroff, K. (2010). The importance of facets of quality of life to older adults: an international investigation. Quality of Life Research, 19(2), 293-298.

34. Molzahn, A., Kalfoss, M., Skevington, S. M., \& Schick Makaroff, K. (2011). Comparing the importance of different aspects of quality of life to older adults across diverse cultures. Age and Ageing, 40(2), 192-199.

35. Snyder, C. F., Blackford, A. L., Aaronson, N. K., Detmar, S. B., Carducci, M. A., Brundage, M. D., et al. (2011). Can patient-reported outcome measures identify cancer patients' most bothersome issues? Journal of Clinical Oncology, 29(9), 1216-1220. 
Figure 1: Example of a graphical profile of WHOQOL-BREF and WHOQOL Importance Physical and Psychological domain scores

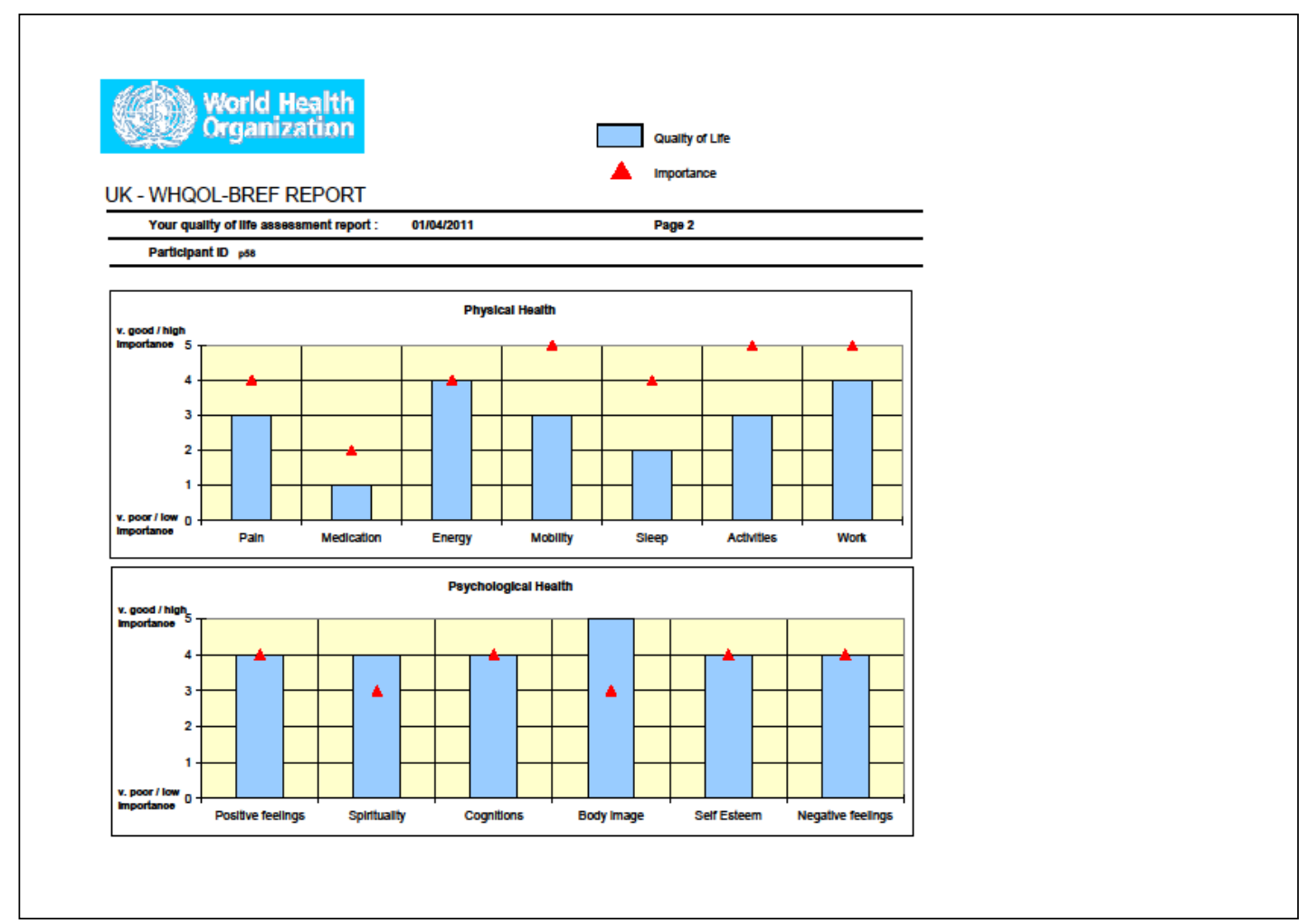


Figure 2: Percentage of respondents reporting Change or No change following feedback

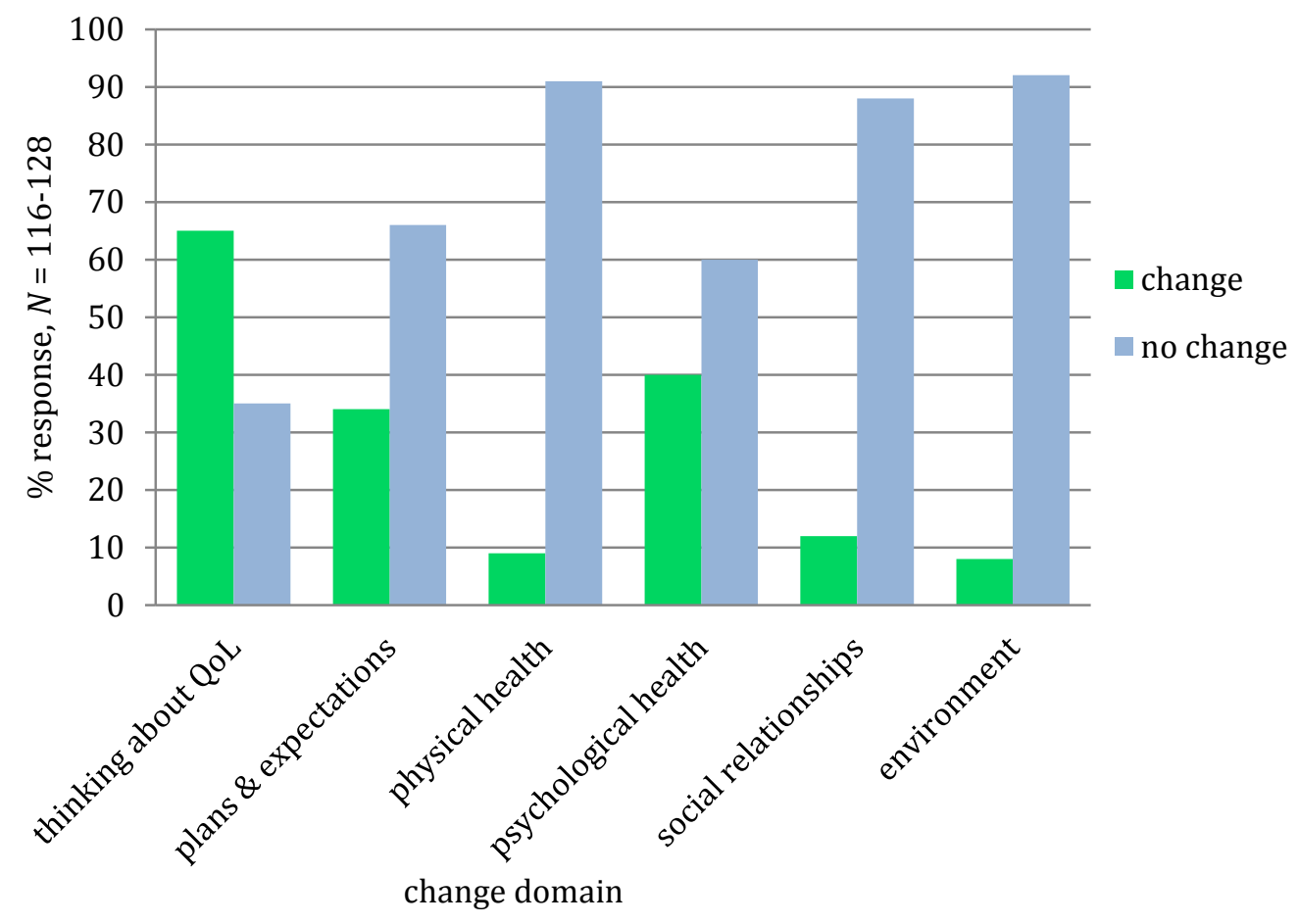


Table 1: Socio-demographic characteristics of the Evaluation sampıe

\begin{tabular}{|c|c|c|c|}
\hline Category & & $N=129$ & $\begin{array}{c}\text { o o of } \\
\text { total } \\
\text { sample }\end{array}$ \\
\hline \multirow[t]{8}{*}{ Age } & $18-24$ & 11 & 8.5 \\
\hline & $24-34$ & 17 & 13.2 \\
\hline & $35-44$ & 18 & 14.0 \\
\hline & $45-54$ & 24 & 18.6 \\
\hline & $55-64$ & 24 & 18.6 \\
\hline & $65-74$ & 19 & 14.7 \\
\hline & $75+$ & 13 & 10.1 \\
\hline & Not disclosed & 4 & 3.1 \\
\hline \multirow[t]{5}{*}{ Education } & Primary & 1 & 0.8 \\
\hline & Secondary & 23 & 18.1 \\
\hline & Technical / Further Education & 21 & 16.5 \\
\hline & College / University & 32 & 25.2 \\
\hline & Post-graduate & 50 & 39.4 \\
\hline \multirow[t]{5}{*}{ Marital status } & Single (never married) & 22 & 17.3 \\
\hline & Married & 66 & 52.0 \\
\hline & Partnered (not married) & 20 & 15.7 \\
\hline & Single / separated / divorced & 10 & 7.9 \\
\hline & Widowed & 9 & 7.1 \\
\hline \multirow[t]{3}{*}{ Living arrangements } & Alone & 25 & 19.8 \\
\hline & With partner / spouse / family & 96 & 76.2 \\
\hline & Other & 5 & 4.0 \\
\hline \multirow[t]{3}{*}{ Location of residence } & Town / city centre & 27 & 21.4 \\
\hline & Suburbs & 60 & 47.6 \\
\hline & Countryside & 39 & 31.0 \\
\hline \multirow[t]{9}{*}{ Employment status } & Employed full time & 43 & 33.9 \\
\hline & Employed part-time & 15 & 11.8 \\
\hline & Self-employed & 8 & 6.3 \\
\hline & Unemployed, looking for work & 3 & 2.4 \\
\hline & Retired & 35 & 27.6 \\
\hline & Unemployed, unable to work & 3 & 2.3 \\
\hline & Student & 10 & 7.9 \\
\hline & $\begin{array}{l}\text { Household duties, carer, voluntary } \\
\text { work }\end{array}$ & 7 & 5.4 \\
\hline & Other & 3 & 2.4 \\
\hline
\end{tabular}


Table 2: Responses to the question "How helpful will your experience of this study be to you in the future?

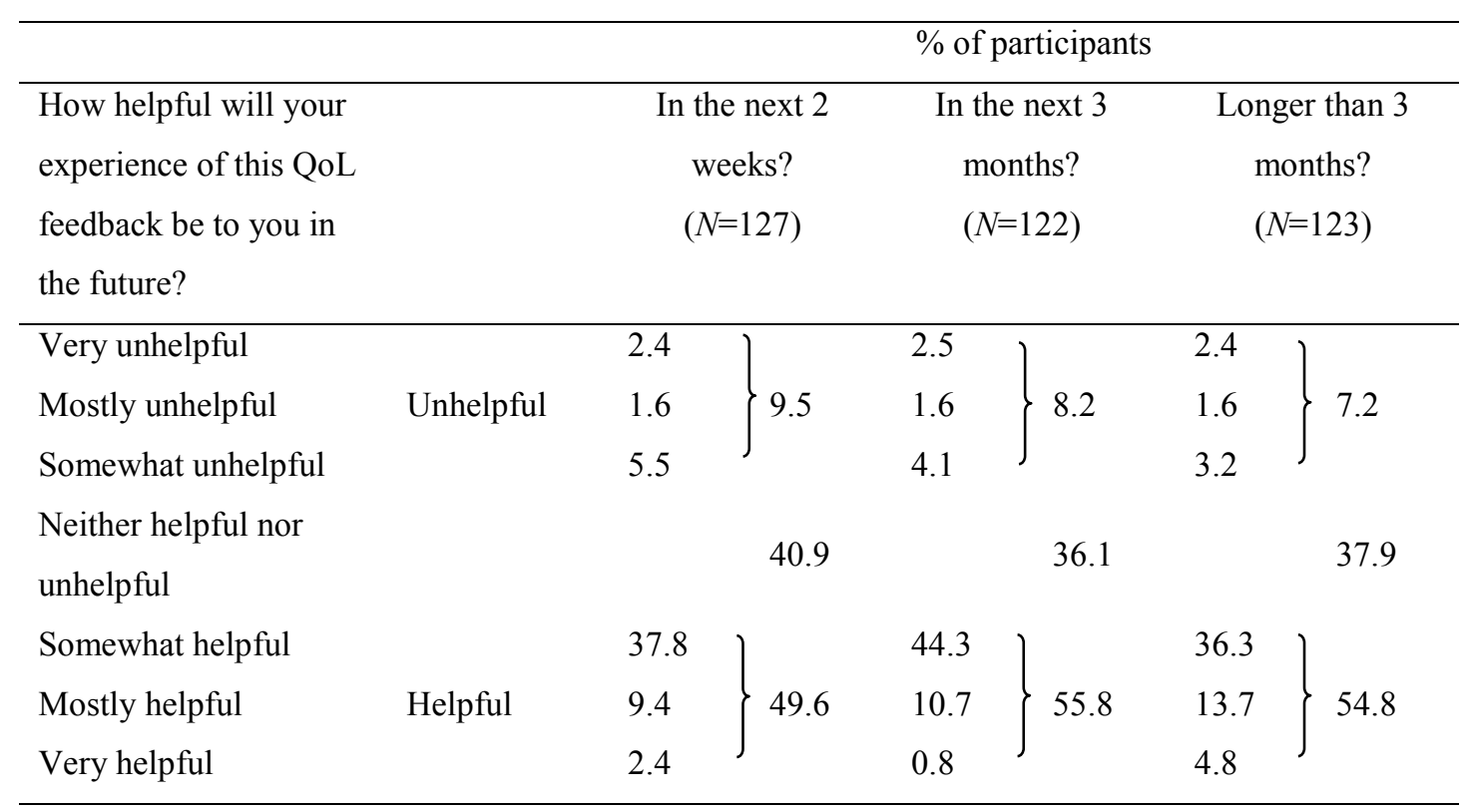

